\title{
A Way to Set up Security Layer over Internet
}

\author{
Xiangyi Hu, Guifen Zhao, Guanning Xu \\ Beijing Key Laboratory of Network Cryptography Authentication, Beijing Municipal Institute of Science \& \\ Technology Information, Beijing, China \\ Email: gfzh@hotmail.com
}

Received March 2015

\begin{abstract}
A security architecture using secret key algorithm and vertical authentication mode is proposed. Establish security protocols in the chip of smart key at network client or mobile phone, and establish key exchange protocol in the chip of encryption cards at network key management center. A combined key real-time generation algorithm is used to solve the update and management problems. Online or offline authentication and documents encryption transmission protocols are adopted to achieve credible connection between users. Accordingly, set up security layer over Internet, which provides convenient encryption ability to each network user, and build credible and secure network system.
\end{abstract}

\section{Keywords}

Security Layer, Vertical Authentication, Combined Secret Key, Credible Network, Online Authentication, Offline Authentication

\section{Introduction}

With the application and popularization of network, network has already widely applied to job, study, daily life, social communication and other fields. Network security always restricts rapid development of network application. It's urgent to solve network security problems [1]-[6], especially the authentication and secure documents exchange problems for mass users. There are hidden troubles in IPv4 network communication protocol. Therefore, it is necessary to build security layer over Internet for users' information security demand [7], especially to master fast key exchange technology. However, if use international third-party authentication security architecture, such as using public key algorithm RSA or ECC establish authentication and data encryption system on Internet, the cost is too high, which can not achieve the purpose to establish security layer over Internet.

We propose a vertical authentication framework shown in Figure 1, using combined key real-time generation algorithm to solve the problem of real-time key exchange, using two authentication mode including online and offline authentication. Establish authentication and documents encryption protocol, and achieve trusted connection between users, that is the real name system of Internet. Every Internet user can carry out simple encryption, personal computer, mobile phone and other equipments are provided with simple and convenient encryption capabilities, which can guarantee user's absolute privacy about e-mail, SMS, WeChat and various data, and avoid APT attack. Thus, set up "security layer” over Internet, and establish trusted network system. 


\section{Security Architecture of Vertical Authentication}

\subsection{Security Architecture at Client}

The proposed security architecture uses smart card at client by way of client encryption system hardware, including USB key or SD key devices.

Establish client encryption system in smart card chip and write secret key cipher algorithm, hash algorithm, combined secret key real-time generation algorithm, offline authentication, online authentication protocol, offline signature and encryption protocol, online signature and encryption protocol, and data including identification $\mathrm{Bi}$ of smart card, a three-dimensional matrix Ti of key seeds, and a three-dimensional matrix $\mathrm{E}$ of another set of key seeds, $i=1-n, n$ is the total number of network users.

Each client smart card owns a unique identification $\mathrm{Bi}$, and different from each other. Corresponding with smart card identification, the three-dimensional matrix Ti of key seeds is different from each other. The elements of Ej stored in user's smart card of the same friends group are the same. $j=1-m, m<n$.

\subsection{Security Architecture at Server}

Set up key exchange center on network which consists of servers and encryption cards. Regard encryption cards as encryption system hardware of key exchange center. Establish server encryption system in encryption card chip and write secret key cipher algorithm, hash algorithm, combined secret key real-time generation algorithm, a three-dimensional matrix D of key seeds, key exchange protocol, and store the cipher text of all users' three-dimensional key seeds matrix Ti in user key data base. Each record in user key data base consists of identification Bi of smart card, $32 \times 2$ matrix Gi composed with digest information of identification Bi and random number $\mathrm{Si}$, cipher text of three-dimensional key seeds matrix Ti, digital signature of three-dimensional key seeds matrix Ti. Specifically, generate storage key Ki according to matrix Gi and three-dimensional matrix D, and then using Ki perform encryption and signature respectively for identification $\mathrm{Bi}$ and elements of three-dimensional matrix Ti. $\mathrm{i}=1-\mathrm{n}, \mathrm{n}$ is the total number of network users.

\section{Key Management for Secret Key Cryptography}

A secret key management technique is proposed to perform key management achieve real-time generation and secure exchange.

\subsection{Procedure Key}

Assume that procedure key is GK. GK is a set of random numbers, 128 bit, generated by random number gene rator of client smart card. Use procedure GK performs signature and encryption for sender user's documents.

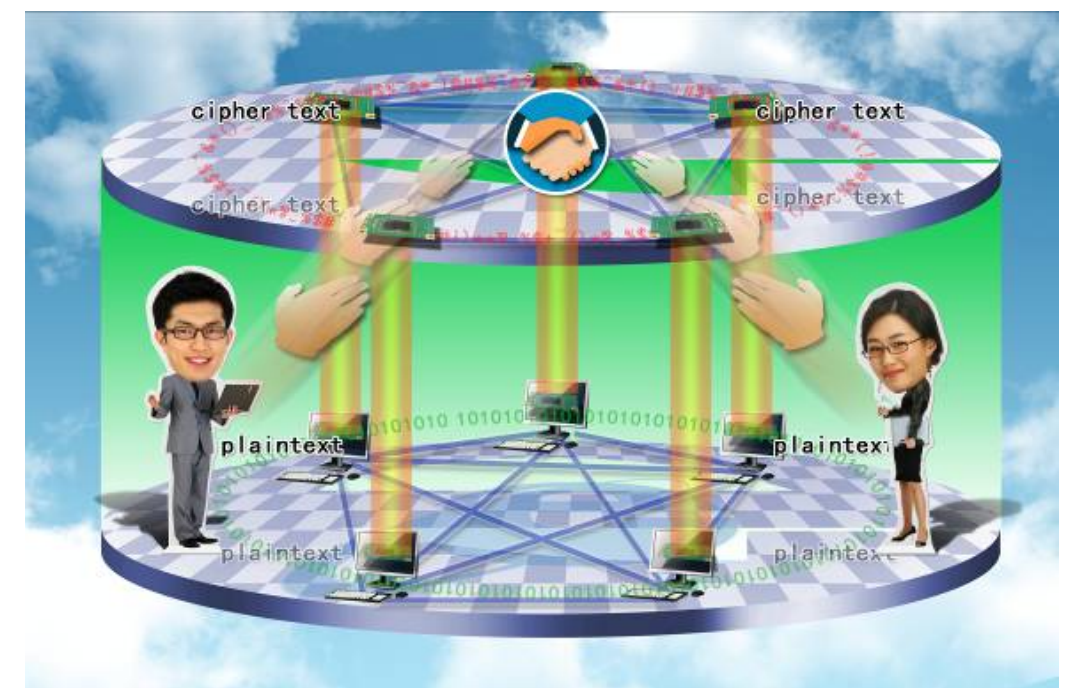

Figure 1. Set up security layer over internet. 
Moreover, use another user key YK encrypt the procedure key GK. The cipher text of GK is GK'. Send GK' to key exchange center and decrypt it, and then use receiver user's key encrypt the plaintext and get a new cipher text. Transmit the new cipher text to receiver. Consequently, two users realize secure exchange of procedure key GK via key management center. Specifically, the plaintext of GK doesn't appear out of encryption card chip of key exchange center.

\subsection{User Key}

Assume that user key is YK. YK is generated by combined key real-time generation algorithm in client smart card. If users are under online environment, user key YK is used to encrypt the procedure key GK, which can achieve secure exchange of procedure key. If users are under offline environment, user key YK is used to perform signature and encryption, and encrypt identifying code to generate signature code within offline authentication protocol. User key adopts centralized generation, centralized writing, and centralized distribution with hardware equipments.

While initializing user key, random number generator of encryption card equipped in key exchange center generate a set of random numbers, and then regard these random numbers as key seeds and constitute a $(32 \times 16$ $\times 16$ ) three-dimensional matrix T. There are 8192 elements in matrix T. Each element is 0.5 byte. Totally the matrix occupies about $4 \mathrm{~KB}$. That is the elements of matrix $\mathrm{T}$ are 4 bit character string. Write the elements of matrix $\mathrm{T}$ into client smart card, and distribute user key with smart card hardware equipments.

Assume that three-dimensional key seeds matrix $\mathrm{T}$ is shown in Figure 2.

While running client authentication protocol or signature protocol, encryption protocol in user's smart card, client encryption system generates a set of user key YK according to combined key real-time generation algorithm on the basis of $(32 \times 2)$ matrix $G$ and three-dimensional key seeds matrix $T$, or generates a set of user key YK on the basis of $(32 \times 2)$ matrix $G$ and three-dimensional key seeds matrix $\mathrm{E}$.

Because different users own different elements of three-dimensional matrix Ti, users can perform offline authentication. Different users own the same three-dimensional matrix E. Users can perform documents encryption transmission.

Random number generator in encryption card quipped at key exchange center generates a set of random numbers, constitutes the elements of $(32 \times 16 \times 16)$ three-dimensional matrix Ej and distributes to all corresponding users within the same friends group. The distribution procedure of $\mathrm{Ej}$ is described: one user among a user group login key exchange center after authentication, and submit other users' identification in the same friends group to key exchange center. The encryption system of key exchange center encrypt the elements of $\mathrm{Ej}$ using different key generated on the basis of corresponding identification Ti in encryption cards. Obtain cipher text of different Ej elements and transfer individually to corresponding client. At each client, encryption system decrypts the cipher text using key generated on the basis of Ti, and writes the plaintext of Ej elements into smart

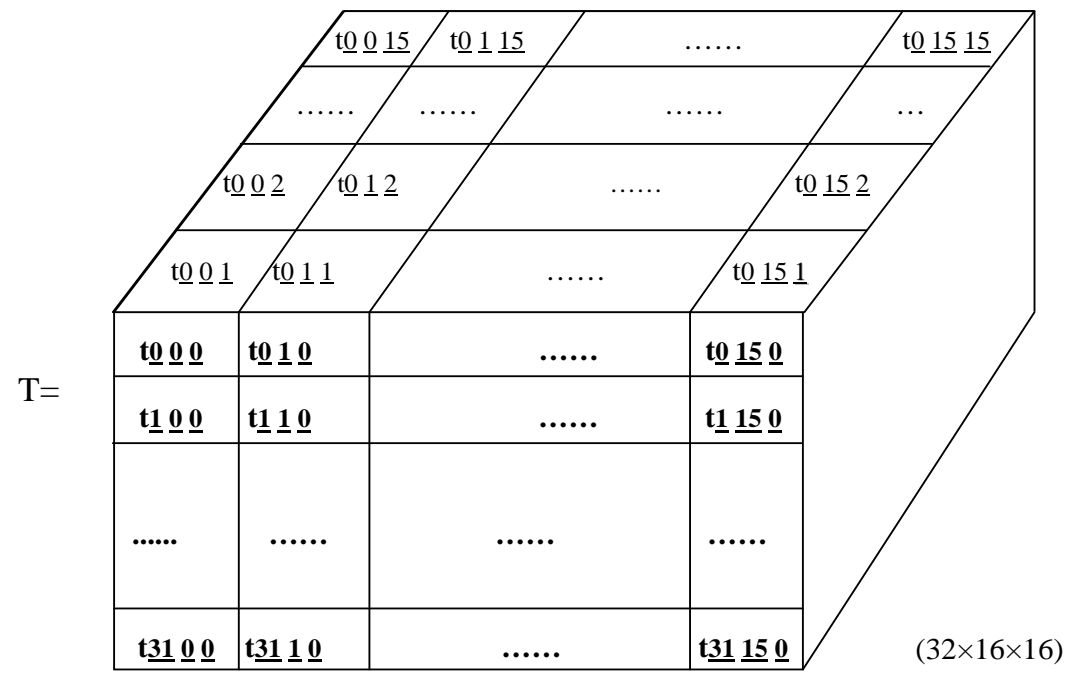

Figure 2. Three-dimensional key seeds matrix T. 
card. $\mathrm{j}=1-\mathrm{m}, \mathrm{m}<\mathrm{n}$.

\subsection{Storage Key}

Assume that storage key is K. K is used to encrypt all users' elements of three-dimensional key seeds matrix T in the encryption cards chip at key exchange center. While key initializing, random number generator in encryption card quipped at key exchange center generates a set of random numbers which are key seeds of key exchange center and constitute the elements of $(32 \times 16 \times 16)$ three-dimensional matrix D. There are 8192 elements in matrix D, and each element is 0.5 byte, totally $4 \mathrm{~KB}$. That is the elements of matrix $\mathrm{D}$ are 4 bit character string. Matrix D is similar with $\mathrm{T}$, but the elements are different.

Write the elements of matrix D into encryption cards of key exchange center. Encryption system of key exchange center generates a set of storage key K according to combined key real-time generation algorithm on the basis of $(32 \times 2)$ matrix GG and three-dimensional key seeds matrix D.

Assume that storage key is K1, K2,.., Kn. Storage key K1, K2,.., Kn encrypt the elements of three-dimensional matrix Ti separately in the encryption cards chip at key exchange center. After encryption, record the cipher text of Ti, corresponding user identification Bi and matrix GGi in user key data base at key exchange center.

Generate storage key Ki on the basis of $(32 \times 2)$ matrix GGi and $(32 \times 16 \times 16)$ three-dimensional matrix D. i $=1-\mathrm{n}, \mathrm{n}$ is the total number of network users.

When cipher text of three-dimensional keys seeds matrix Ti is called at key exchange center, firstly, the cipher text is decrypted in encryption card, and all users' plaintext of three-dimensional key seeds matrix Ti doesn't appear out of encryption card chip. Therefore, guarantee storage and running security of all users' key seeds elements of three-dimensional matrix Ti at key exchange center. $\mathrm{i}=1 \sim \mathrm{n}, \mathrm{n}$ is the total number of network users.

\subsection{Combined Key Real-Time Generation Algorithm}

Combined key real-time generation algorithm means that client smart card identity and the 256 bit hash value of a set of random numbers constitute a $(32 \times 2)$ matrix, for example matrix G or matrix GG. If the matrix is $G$, perform mapping for three-dimensional key seeds matrix T according to elements of $(32 \times 2)$ matrix $\mathrm{G}$. The selected elements of three-dimensional matrix T constitute a user key YK. Similarly, storage key K is generated according to matrix GG and three-dimensional matrix D.

Generate a set of random numbers by random number generator of client smart card. Encryption system client calls hash algorithm to deal with the smart card identification $\mathrm{Bi}$ and the random numbers, and then obtains a set of hash value. The hash value constitutes a $(32 \times 2)$ matrix G. According to combined key real-time generation algorithm, constitute a user key on the basis of $(32 \times 2)$ matrix $G$ and $(32 \times 16 \times 16)$ three-dimensional key seeds matrix T.

Generate a set of random numbers by random number generator of encryption card at key exchange center. Encryption system at key exchange center calls hash algorithm to deal with the smart card identification Bi and the random numbers, and then obtains a set of hash value. The hash value constitutes a $(32 \times 2)$ matrix GG. According to combined key real-time generation algorithm, constitute a storage key on the basis of $(32 \times 2)$ matrix GG and $(32 \times 16 \times 16)$ three-dimensional key seeds matrix D. Storage key is applied to encrypt the smart card identification $\mathrm{Bi}$ and corresponding $(32 \times 16 \times 16)$ three-dimensional key seeds matrix $\mathrm{T}$.

Specifically, combined key real-time generation algorithm is that calls hash algorithm, for example SHA-1, SM3, etc. to deal with the smart card identification $\mathrm{Bi}$ and random numbers $\mathrm{Si}$, and then obtains a set of 256 bit hash value. Divide the 256 bit hash value into 64 groups and each group is 4 bit, consequently, constitute a (32 $\times$ 2) matrix. Assume that $(32 \times 2)$ matrix comprised with 256 bit hash value of a set of random numbers is $\mathrm{G}$.

$$
G=\left(\begin{array}{cc}
g_{00} & g_{01} \\
g_{10} & g_{11} \\
\vdots & \vdots \\
g_{310} & g_{311}
\end{array}\right)_{(32 \times 2)}
$$

The total number of matrix $\mathrm{G}$ elements is $0-15$.

The first row and first column element of matrix $G$ is $g_{0} 0$, mapping the first row and $g_{0}$ column elements of 
$\mathrm{T}$. The first row and second column element of matrix $\mathrm{G}$ is $\mathrm{g}_{0}$, mapping the first row, $\mathrm{g}_{0} 0$ column and $\mathrm{g}_{01}$ page element of T. The element is selected and assumed it is kk1.

Similarly, the second row and first column element of matrix $G$ is $g_{10}$, mapping the second row and $g_{10}$ column elements of $T$. The second row and second column element of matrix $G$ is $g_{11}$, mapping the second row, $g_{1}$ ${ }_{0}$ column and $\mathrm{g}_{11}$ page element of $\mathrm{T}$. The element is selected and assumed it is kk2.

Until the thirty-second row, $\mathrm{g}_{310}$ column and $\mathrm{g}_{311}$ page element of $\mathrm{T}$ is selected and assumed it is kk32.

The kk1, kk2,..., kk32 compose a set of key which is user key or storage key.

\subsection{Procedure Key GK, User Key YK and Storage Key K}

Procedure key GK, user key YK and storage key K are all 128 bit. Repetition probability of GK is $1 / 2^{128}$. Basically, GK is one-time.

User key and storage key are generated by combined key real-time generation algorithm. That is mapping three-dimensional matrix T according to 64 elements of $(32 \times 2)$ matrix G. The selected elements of three-dimensional matrix T constitute a user key YK. There are 32 rows and 2 columns in matrix G, and each element is a number between $0-15$, totally 16 varieties, therefore, there are 2 elements and $16 \times 16=2^{8}$ varieties in each row. For total 32 rows, the key variation is $2^{(8 \times 32)}=2^{256}$. Accordingly, the key generated by combined key real-time generation algorithm, including user key and storage key, is basically one-time and not repeated.

\section{Offline Security Protocol}

While performing offline authentication between user 1 and user 2, both clients call the same three-dimensional key seeds matrix E stored in smart card to generate user key. Key exchange center is not needed while user key exchanging.

\subsection{Offline Authentication Protocol}

Firstly, client encryption system of user 1 generates a set of random numbers $\mathrm{S}$ in smart card. The hash value of identification B1 and random number S is identifying code L1, and constitutes matrix GG. User key YK1 is created according to matrix GG and three-dimensional matrix E, and applied to encrypt identifying code L1. The cipher text of L1 is signature code L1'. Client encryption system of user 1 sends the identification B1, identifying code L1 and signature code L1' to user 2. The client encryption system of user 2 generates symmetric key YK2 according to matrix GG and three-dimensional matrix E in smart card, and decrypts signature code L1' to obtain identifying code L2. Compare identifying code L1 and L2 for identification. If L1 $\neq$ L2, user 1 is illegal user, otherwise L1 = L2, user's identity is true. Similarly verify the identification of user 2 according to the same protocol. Thereby, offline authentication between user 1 and user 2 is achieved [8].

\subsection{Offline Signature and Encryption Protocol}

Firstly, client encryption system of user 1 generates a set of random numbers $\mathrm{S}$ in smart card. The hash value of identification B1 and random number S is identifying code L1, and constitutes matrix GG. User key YK1 is created according to matrix GG and three-dimensional matrix E, and applied to encrypt document 1 and its digital digest M1, and then get the cipher text and digital signature of document 1. Client encryption system of user 1 sends the identification B1, matrix GG, the cipher text, digital digest, and digital signature of document 1 to user 2.

The client encryption system of user 2 generates symmetric key YK2 according to matrix GG and three-dimensional matrix E in smart card, and decrypts the cipher text and digital signature of document 1 to obtain the plaintext and digital digest M2 of document 1. Compare digital digest M1 and M2 and validate integrity and credible. If $\mathrm{M} 1 \neq \mathrm{M} 2$, document 1 has been tampered, otherwise $\mathrm{M} 1=\mathrm{M} 2$, document 1 is integrate and credible. Thereby, offline cipher text transmission and integrity verification of document 1 between user 1 and user 2 is achieved.

\section{Online Security Protocol}

While performing online authentication between user 1 and user 2, both clients call different three-dimensional 
key seeds matrix T1 and T2 stored in respective smart card to generate user key. Key exchange center is needed while user key exchanging.

\subsection{Online Authentication Protocol}

Firstly, client encryption system of user 1 generates a set of random numbers S1 and S2 in smart card. S2 is the procedure key GK1. The hash value of identification B1 and random number S1 is identifying code L1, and constitutes matrix G. User key YK1 is created according to matrix G and three-dimensional matrix T1, and applied to encrypt GK1. The cipher text of GK1 is GK1'. The GK1 is applied to encrypt identifying code L1. The cipher text of L1 is signature code L1'. Client encryption system of user 1 sends the identification B1, identification B2, identifying code L1, signature code L1' and GK1' to key exchange center.

The encryption system at key exchange center generates storage key K1 according to identification B1 to decrypt the cipher text of T1 and perform integrity verification. Generate user key YK-1 according to matrix G and T1 to decrypt the cipher text GK1'. And then generate storage key K2 according to identification B2 to decrypt the cipher text of T2 and perform integrity verification. The hash value of identification B2 and random number S1 is identifying code LL, and constitutes matrix GL. User key YK2 is created according to matrix GL and three-dimensional matrix T2, and applied to encrypt GK1. Encryption system of key exchange center sends the identification B1, identification B2, matrix GL, identifying code L1, signature code L1' and cipher text of GK1 to user 2.

The client encryption system of user 2 generates symmetric key YK-2 according to matrix GL and three-dimensional matrix T2 in smart card, and decrypts signature code L1' to obtain identifying code L2. Compare identifying code L1 and L2 for identification. If $\mathrm{L} 1 \neq \mathrm{L} 2$, user 1 is illegal user, otherwise $\mathrm{L} 1=\mathrm{L} 2$, user is true user. Similarly verify the identification of user 2 according to the same protocol. Thereby, online authentication between user 1 and user 2 is achieved.

\subsection{Online Signature and Encryption Protocol}

Firstly, client encryption system of user 1 generates a set of random numbers S1 and S2 in smart card. S2 is the procedure key GK1. The hash value of identification B1 and random number S1 is identifying code L1, and constitutes matrix G. User key YK1 is created according to matrix G and three-dimensional matrix T1, and applied to encrypt GK1. The cipher text of GK1 is GK1'. The GK1 is applied to encrypt document 1 and the digital digest M1 of document 1 , and then obtain the cipher text and digital signature of document 1 . Client encryption system of user 1 sends the identification B1, identification B2, matrix G, cipher text and digital signature of document 1 , and GK1' to key exchange center.

The encryption system at key exchange center generates storage key K1 according to identification B1 to decrypt the cipher text of T1 and perform integrity verification. Generate user key YK-1 according to matrix G and T1 to decrypt the cipher text GK1'. And then generate storage key K2 according to identification B2 to decrypt the cipher text of T2 and perform integrity verification. The hash value of identification B2 and random number S1 is identifying code LL, and constitutes matrix GL. User key YK2 is created according to matrix GL and three-dimensional matrix T2, and applied to encrypt GK1. Encryption system of key exchange center sends the identification B1, identification B2, matrix GL, the cipher text and digital signature of document 1 , and cipher text of GK1 to user 2.

The client encryption system of user 2 generates symmetric key YK-2 according to matrix GL and three-di-mensional matrix T2 in smart card, and decrypts the cipher text and digital signature of document 1 to obtain the plaintext and digital digest M2 of document 1. Compare digital digest M1 and M2 in smart card of user 2 client. If $\mathrm{M} 1 \neq \mathrm{M} 2$, document 1 has been tampered, otherwise M1 = M2, document 1 is integrate and credible. Thereby, online cipher text transmission and integrity verification of document 1 between user 1 and user 2 is achieved.

\section{Advantages of Vertical Authentication}

\subsection{Characteristics of PKI}

PKI uses third-party authentication infrastructure. The characteristic of signature and encryption protocol is that two cipher modes are adopted simultaneously to establish digital signature and encryption protocol, i.e. symmetric algorithm and asymmetric algorithm. Illustration is shown in Figure $\mathbf{3}$ and described as follow: 
- The random number generated by PKI is the session key K.

- Use AES (or 3DES) and K to encrypt data and obtain cipher text.

- Use RSA (or ECC) and user's private key to perform digital signature.

- Use RSA (or ECC) and the public key of CA to encrypt the session key K.

\subsection{Characteristics of Vertical Authentication Architecture}

The characteristic of signature and encryption protocol used in vertical authentication architecture is that only one symmetric cipher algorithm is adopted to establish signature and encryption protocol, shown in Figure 4. The security architecture is simple, only a set of key is used while performing data encryption and signature.

Generate secret key K according to the combined secret key algorithm, and then exchange the K via random numbers and time-stamp.

- Use AES (or 3DES) and K to perform digital signature and encrypt data to obtain cipher text.

\subsection{Comparison between PKI and Vertical Authentication Architecture}

Compare the signature and encryption protocol of PKI third-party authentication and vertical authentication architecture. It is obvious that PKI calls 2 more asymmetric cryptography algorithms and 2 public key. Use public key algorithm performs twice encryption, one is encryption of digital digest and another is encryption of procedure key for key exchange. The vertical authentication architecture doesn't call 2 asymmetric cryptography algorithms and 2 public key. Symmetric cryptography algorithm runs 100 times in computer and 1000 times in

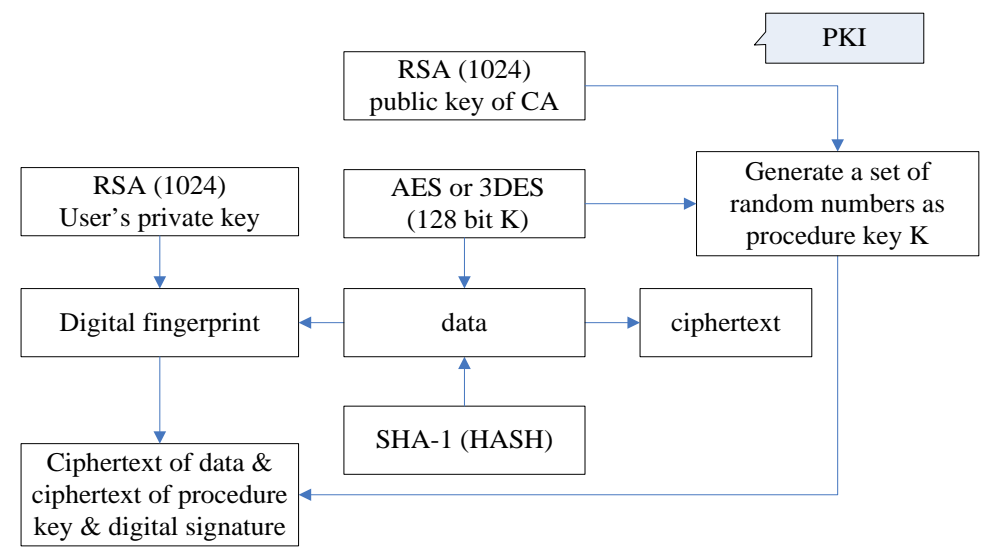

Figure 3. Flow Chart of PKI signature and encryption protocol.

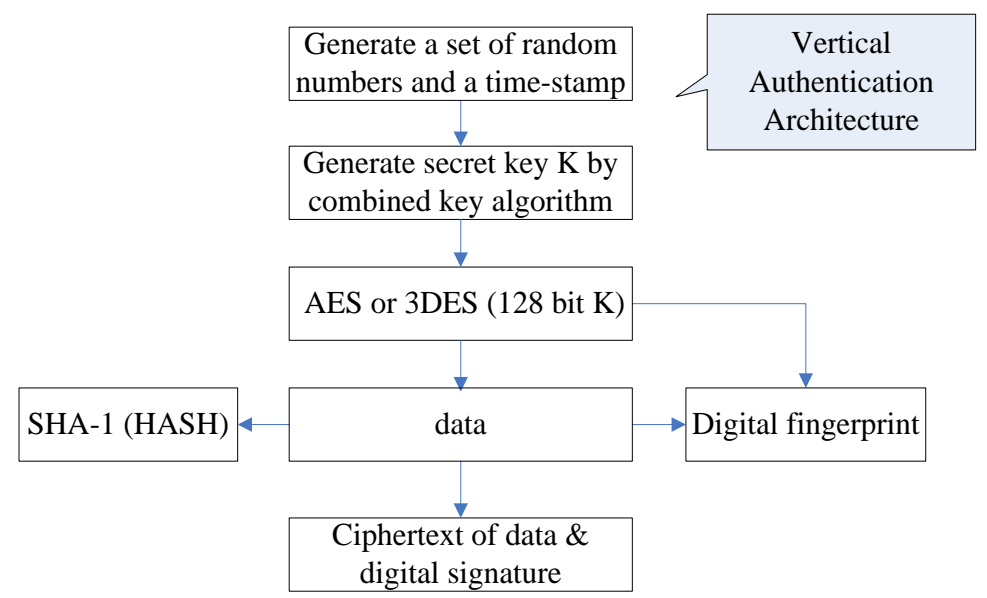

Figure 4. Flow chart of signature and encryption protocol used in vertical authentication architecture. 
chipsets faster than asymmetric cryptography algorithm. That is why this method runs faster and high-efficiency than PKI.

\section{Conclusion}

The vertical authentication architecture is proposed using centralized key generation, initialization and distribution via hardware equipments. Combined key real-time generation algorithm is applied to solve the problems of update and management. Online and offline authentication protocol can achieve credible connection between users and realize real name net play. Set up security layer over Internet, which provides convenient encryption ability for personal computer, mobile phone and other communication equipments of each network user, and achieve encryption transmission between users. The method can avoid APT attack and guarantee domestic network system secure and credible.

\section{Acknowledgements}

The work is supported by Innovation Project II-2: Research and Development of Cryptographic Authentication System in Cloud Computing Security (No. PXM2014_178214_000011), Beijing Key Laboratory of Network Cryptography Authentication, Beijing Municipal Institute of Science \& Technology Information.

\section{References}

[1] Bai, J. and Jing, J.W. (2012) Look upon the Cryptography Challenge in Cloud. China Information Security, 11, 15-16, 26.

[2] Shen, C.X. (2012) Cloud Computing Security and Classified Security Protection. China Information Security, 1, 16-17.

[3] Han, S. (2012) Key Technologies on Cloud Computing Data Security. University of Electronic Science and Technology of China.

[4] Feng, D.G. (2011) Enter Cloud Computing Security Age. Netinfo Security, 3, 1-2.

[5] Zhang, W.-K. and Liu, G.-F. (2012) Data Security and Privacy Protection of Cloud Computting. China Information Security, 11, 38-40.

[6] Zhang, Y.Y., Chen, Q.J., Pan, S.B. and Wei, J.W. (2010) Key Security Technologies on Cloud Computing. Telecommunications Science, 26, 64-69.

[7] Online Privacy Breakthrough as British Researchers Claim to Have Developed Way to Give the Internet a "Security Layer".

http://www.dailymail.co.uk/sciencetech/article-2885677/Online-privacy-breakthrough-British-researchers-claim-devel oped-way-internet-security-layer.html?ITO=1490\&ns_mchannel=rss\&ns_campaign=1490

[8] Hu, X.Y. (2014) Patent Title: A Method of Mobile Phone Offline Authentication. Patent Application Number: 201410833988.X. 